\title{
Aspect Ratios of Historical Societies and Their Relation To Climate
}

\author{
Christopher Wolfram
}

\begin{abstract}
Jared Diamond proposed that societies grow in accordance with regional differences in climate, and that because climate varies more drastically when moving north-south than when moving east-west, large societies will grow faster east-west than north-south. We characterize aspect ratio for regions on the globe and find that societies are on average wider than they are tall, supporting Diamond's claim. However, we find a similar effect with small and large societies, suggesting another underlying cause. We then investigate the first part of Diamond's claim, finding that societies do not grow in accordance with climate in the manner he predicts.
\end{abstract}

\section{Introduction}

In his book, Guns, Germs, and Steel [1], Jared Diamond proposes that the axes of continents had, and perhaps still have, an influence on the spread of agriculture and indirectly on the spread of technology, ideas, and societies. Diamond argues that domesticated crops and livestock will often be best suited for the climate in which they were domesticated, guiding the spread of these domesticates into other regions of similar climate and away from regions with significantly different climates. He then proposes that because climate generally changes more drastically with latitude than it does with longitude, these domesticates will generally spread more easily east-west than they will north-south. The constraints of agriculture, he says, can then influence the spread of technology and the growth of empires.

Though data regarding the spread of agriculture and technology is scarce, much more data exists on the spread of societies. Mathematica provides information on the borders of about a thousand historical and modern societies. Most of this data was originally collected as part of the GeaCron Project (http://www.geacron.com). This data likely contains many small errors. However, we only care about the general shape of each society, and so these small errors should not be significant for us. In addition, it is unlikely that these errors have any particular directional bias, and so given the amount of data, the errors should roughly cancel themselves out.

Some work has already been done on Diamond's second claim that societies bias toward eastwest growth, but it has either been done with small sample sizes or without fully systematic methods for characterizing the growth of the sampled societies [2,3]. In addition, little work has been done on Diamond's first claim that societies grow so as to minimize the diversity of climates within their borders. Both of these will be investigated here, starting with the question of whether societies bias toward east-west growth.

\section{The East-West Bias of Societies}

\subsection{Characterizing Aspect Ratio}

We can restate Diamond's claim in terms of aspect ratios. Diamond argues that societies on average will be wider than they are tall, meaning that they will have an aspect ratio greater than 1 . In 
order to test this claim, we need a way of measuring the aspect ratios of every society for which we have data.

\subsubsection{Bounding Regions}

There are a few intuitive characterizations of aspect ratio that we can start with. We might imagine drawing the society in question on a map, measuring its width and height, and dividing appropriately. However, that description ignores the important step of projecting the society from the globe onto a map using some map projection. This method, and a number of similar methods, is projection dependent, meaning that it will give us a different answer depending on the projection we choose. In fact, in extreme cases we can get opposite results by choosing different projections (Figure 1). Because map projections skew the data to give us virtually any aspect ratio, we cannot rely on any map projection to characterize the aspect ratio of a region on the globe. Instead, we need some projection-independent characterization.

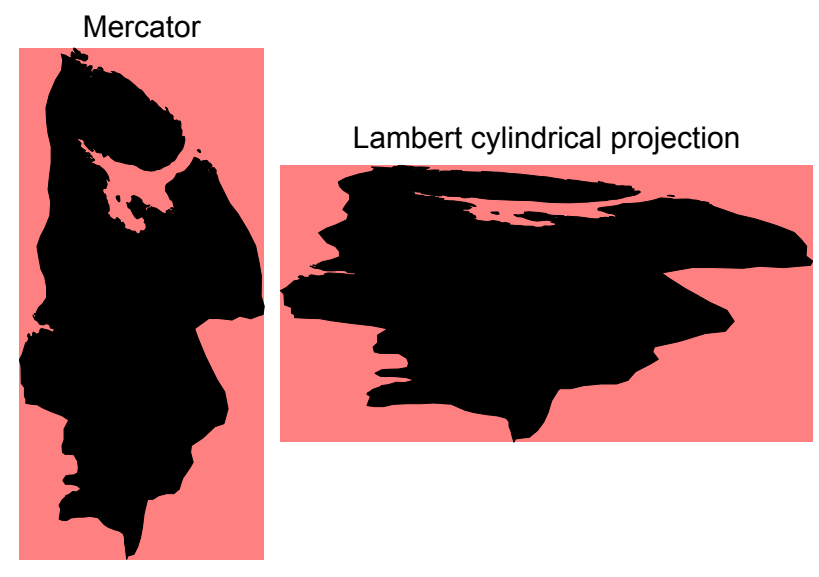

Figure 1: The Tsardom of Russia in 1548 using the Mercator projection (left), and a Lambert cylindrical projection (right). The black regions show the borders of the Tsardom of Russia while the red regions show the bounding rectangles in each projection. We can see that even though the black regions represent the same region on the globe, they have significantly different aspect ratios after being projected. In fact, the aspect ratio of the Mercator projected region is about 1:2, while the aspect ratio of the Lambert cylindrical projected region is about 2:1.

\subsubsection{Bounding Coordinates}

Perhaps the simplest projection-independent characterization of aspect ratio is based on bounding latitudes and longitudes. For some region on the globe, we can measure its height and width by calculating the difference between its minimum and maximum latitudes and longitudes. However, we cannot directly compare the difference in minimum and maximum latitudes to the corresponding difference in longitudes because degrees latitude and degrees longitude are not compatible units. While $1^{\circ}$ latitude represents roughly the same distance everywhere on the globe (just over $110 \mathrm{~km}$ ), $1^{\circ}$ longitude represents different distances depending on the latitude (ranging from $0 \mathrm{~km}$ at the poles to about $110 \mathrm{~km}$ at the equator).

To make our measurements of height and width compatible, we would need to convert the degrees latitude and longitude into units of distance. Figure 2 shows one method for doing this. Because degrees latitude convert to units of distance relatively consistently, it is not hard to convert 
the bounding latitudes into a measure of height. In order to measure width, we choose two points at the correct longitudes and at some characteristic latitude (generally the mean of the minimum and maximum latitudes) and measure the distance between them along lines of constant latitude.
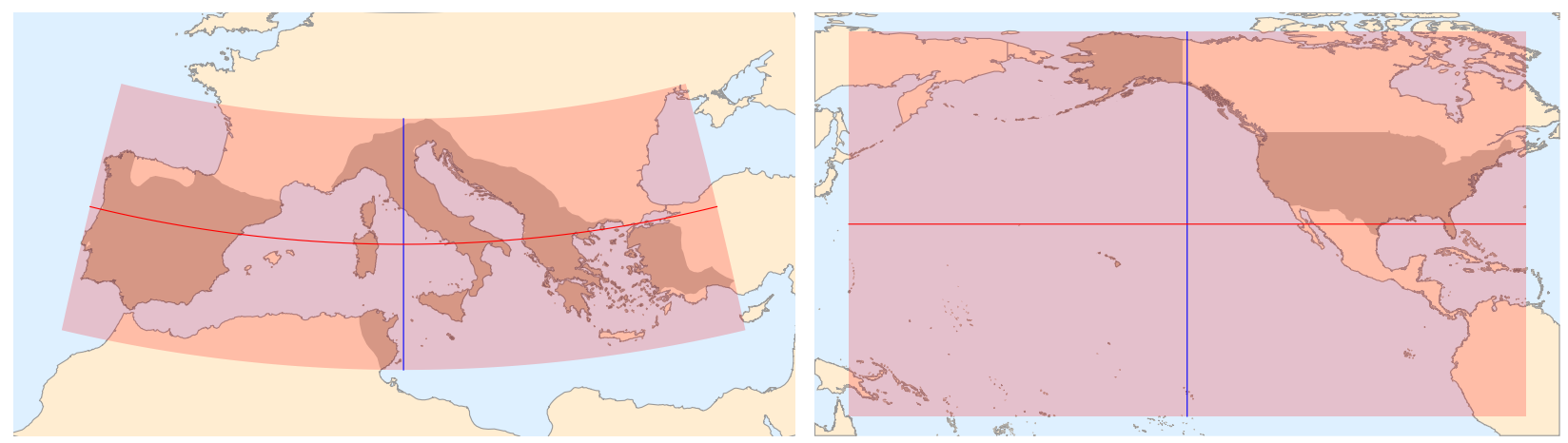

Figure 2: The left shows the bounding coordinates of the Roman Republic in the year 129 BCE. The right shows the bounding coordinates of the modern United States. The red-tinted regions show the bounding latitudes and longitudes. The length of the blue lines is taken as the measure of height and the length of the red lines is taken as the measure of width.

There are two problems with this method. First, our choice of latitude when measuring the width can give us systematically skewed results. Here, we arbitrarily chose the mean latitude, but if instead we chose the minimum or maximum latitude we could systematically measure each society as being wider or narrower than we would otherwise. Second, because this method is based on bounding coordinates, it is extremely sensitive to outliers, as has been noted before [2]. To illustrate this, Figure 2 shows the bounding coordinates of the modern United States. The 48 contiguous states have no effect on this bounding region because the extreme points of the United States lie in the US Virgin Islands (in the east), Guam (in the west), Alaska (in the north), and American Samoa (in the south). As a result, the bounding coordinates of the United States mostly contain the Pacific Ocean, and do not represent the shape of the majority of the country.

We need a characterization of aspect ratio that is not only projection independent, but also insensitive to outliers.

\subsubsection{Moments of Inertia}

We can measure the width and height of each society by measuring its moment of inertia around certain axes in 3D. That is, for some society, we start by taking its unprojected shape in 3D (following the curvature of the Earth). We can then measure its width by calculating its moment of inertia around a vector pointing north or south and its height by calculating its moment of inertia around a vector pointing east or west (see Figure 3). Let $\mathbf{v}_{c}$ be the vector from the center of the Earth to the society's center of mass, and let $\mathbf{v}_{p}$ be the vector from the center of the Earth to the North Pole. We can calculate the vector $\mathbf{v}_{w}$ that points west from the society's center of mass by evaluating $\mathbf{v}_{w}=\mathbf{v}_{c} \times \mathbf{v}_{p}$, and we can calculate the vector $\mathbf{v}_{s}$ that points south from the society's center of mass by evaluating $\mathbf{v}_{s}=\mathbf{v}_{c} \times \mathbf{v}_{w}$. The society's moment of inertia around $\mathbf{v}_{s}$ will be our measure of width and its moment of inertia around $\mathbf{v}_{w}$ will be our measure of its height.

Now that we have a measure of width and height, we can define the aspect ratio as the width divided by the height. In many visualizations and calculations, we will look at the log of the aspect ratio. We take the log so that defining the aspect ratio as the height over the width instead of the width over the height is reduced to a difference in sign. We will often average using the geometric 


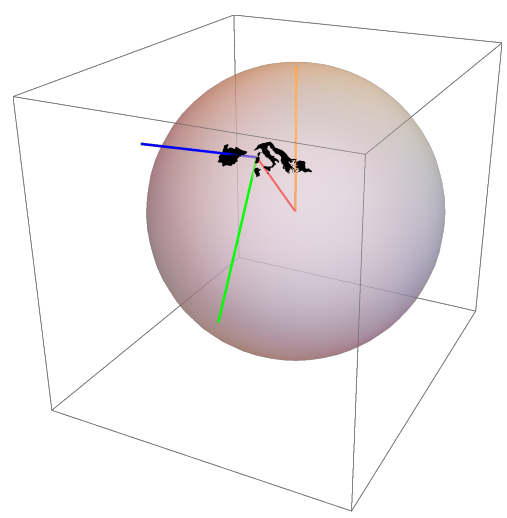

Figure 3: The globe represents the Earth, with the curved black region representing the borders of the Roman Republic in $129 \mathrm{BCE}$. The orange line represents the direction of $\mathbf{v}_{p}$, the red line represents the direction of $\mathbf{v}_{c}$, the blue line represents the direction of $\mathbf{v}_{w}$, and the green line represents the direction of $\mathbf{v}_{s} \cdot \mathbf{v}_{s}$ and $\mathbf{v}_{w}$ point south and west respectively along the surface of the Earth from the center of the Roman Republic.

mean of the aspect ratios because it is equal to the exponential of the arithmetic mean of the log of the aspect ratios.

\section{$2.2 \quad$ Results}

We can now calculate the aspect ratio of each border shape in our dataset. We should note that Mathematica often provides border shapes at multiple points in time for a single society, but for much of our analysis we will only consider each society at its largest extent. Otherwise we would give societies for which we have more detailed data more weight than societies for which we have less detailed data. For example, Mathematica provides 63 border shapes for the Roman Empire but only 1 for Albania, but we do not want to give the Roman Empire 63 times more weight than Albania.

We can calculate that the geometric mean of the aspect ratios is about 1.11. Because the geometric mean is greater than $1\left(p \approx 10^{-5}\right)$, we can see that societies are on average wider than they are tall, supporting Diamond's claim.
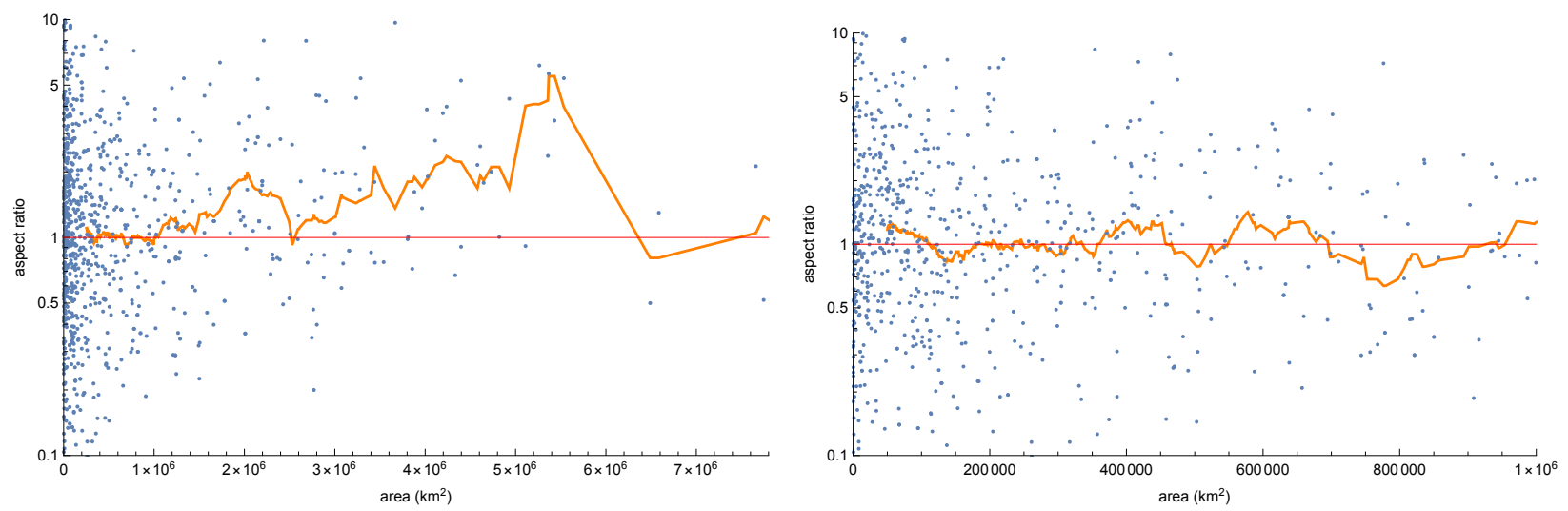

Figure 4: Aspect ratio of societies as a function of area. Aspect ratio is shown on a log scale. Each blue point represents a society at its largest extent. The horizontal red line marks an aspect ratio of 1 . Societies that are wide appear above the line, while those that are tall appear below it. The orange curve shows the moving geometric mean with span $500000 \mathrm{~km}^{2}$. The plot on the right has a smaller domain on the x-axis and uses a moving average with span $100000 \mathrm{~km}^{2}$.

Diamond's argument is only supposed to apply to very large societies, so larger societies should exhibit a larger effect. Figure 4 shows the aspect ratio of each society as a function of its area. 
We can see that many relatively small societies seem to exhibit very little effect, while larger ones exhibit a small and unreliable effect. The lack of a reliable upward slope and the lack of a large difference in effect size between small and large societies suggest that there could be other, less area-dependent factors that drive societies to grow horizontally.

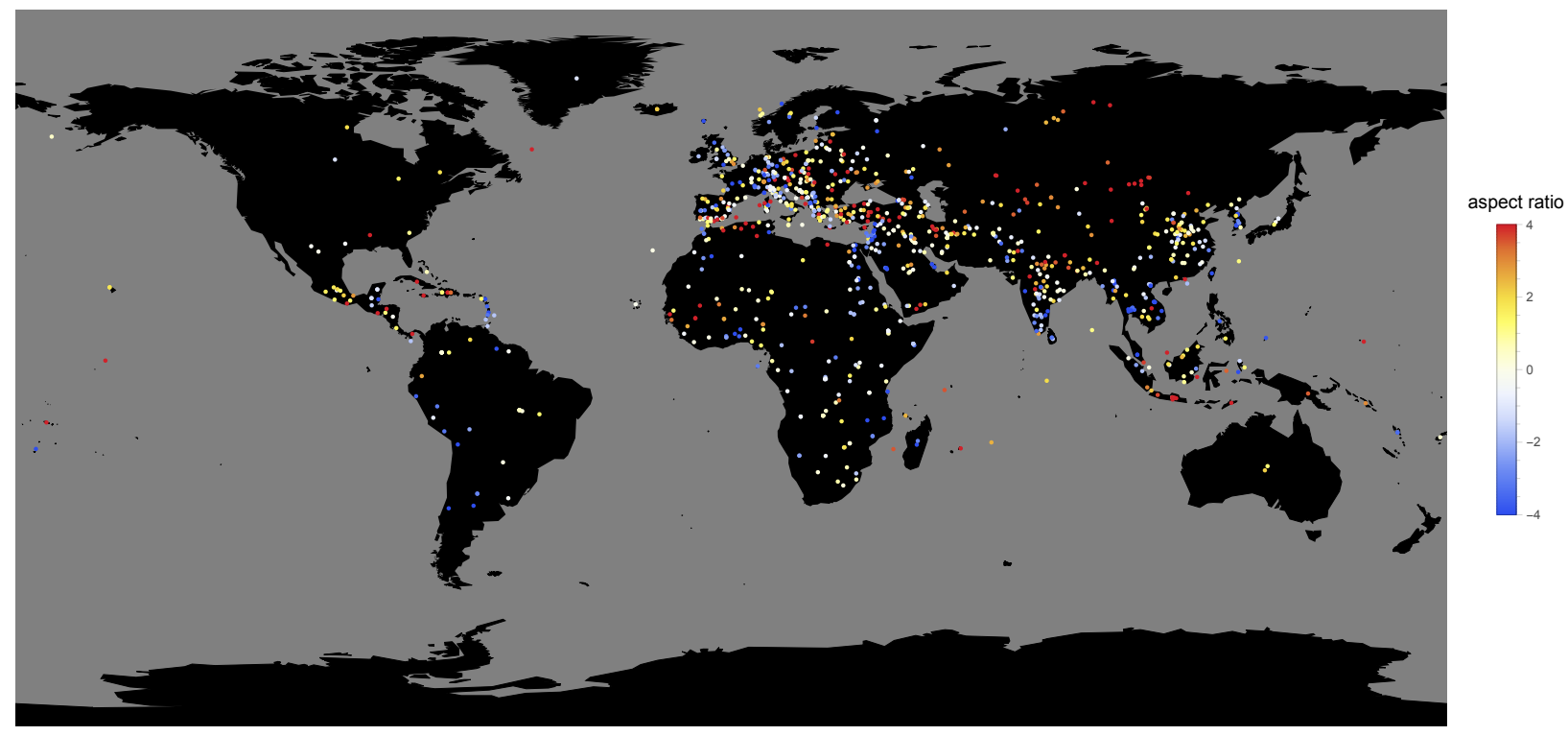

Figure 5: Each disk is placed approximately at the center of each society, and its color represents its aspect ratio. Red disks represent wide societies while blue disks represent tall ones. White disks are neither wide nor tall.

In order to understand why societies have the aspect ratios that they do, it would help to see not just how area affects aspect ratio, but also geography. Figure 5 shows how aspect ratios are distributed across the globe. The orientation of nearby coasts appears to have a large effect on the society's aspect ratio. For example, societies in Asia Minor are mostly wide, reflecting the horizontal coasts, while societies in northern Italy and southern India are taller, reflecting the vertical coasts. It is possible that we have more data in regions with horizontal coasts, creating a selection bias for more horizontal societies.

At this point, we can see that the societies about which we have data are generally wide, which seems to validate Diamond's claim. However, it is harder to see if there are other, larger effects (such as the orientation of coastlines) that might overshadow the climatological effect that Diamond proposes.

\section{Societal Growth and Climate}

Now that we have investigated Diamond's claim about the aspect ratios of societies, we should turn to investigate his underlying claim about the effect of climate on societal growth. Diamond argued that societies grow more easily into regions with a similar climate to their own, implying that societies should have relatively uniform climates. Therefore, we will begin by characterizing climate diversity. 


\subsection{Characterizing Climate Diversity}

There are a number of different variables that we could use in characterizing climate. In his discussion of societal growth, Diamond focuses on temperature, rainfall, and vegetation. Here, we will also consider temperature and rainfall, and we will use primary productivity as a basic measure of the amount of vegetation [4].

Because we do not have high-resolution historical climate data, we are restricted to using modern data. Though the modern climate may not perfectly reflect the historical climate, we will generally look at the variance, and so uniform shifts toward more or less rain, or higher or lower temperatures, should not affect our analysis of climate diversity.

We can start by taking monthly temperature, primary productivity, and rainfall data from NASA NEO (https://neo.sci.gsfc.nasa.gov). We can combine the monthly data at each point into 12-dimensional vectors representing each climate variable over the 12 months of the year.

In order to calculate the temperature diversity of a given region on the Earth, we will collect all the 12-dimensional vectors that lie within that region, concatenate them into a single large list, and then calculate standard deviation of all the elements of that list. Essentially, we calculate the standard deviation of all temperatures that appear within the region at any time of year. For example, Figure 6 shows an approximation of the distribution of temperatures that now appear in the region that the Roman Republic covered in $129 \mathrm{BCE}$. The standard deviation of this distribution is what we use as the temperature diversity of Rome in 129 BCE.

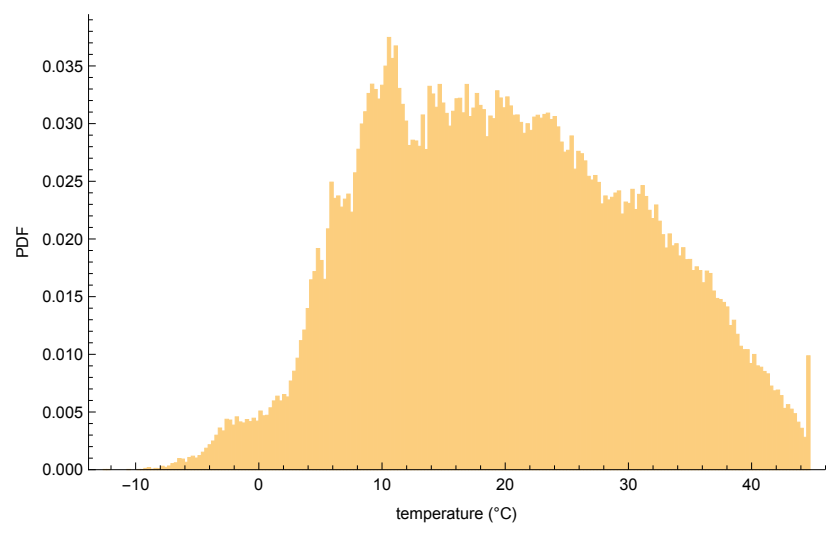

Figure 6: The year-round modern distribution of temperatures in the region of the Roman Republic in the year 129 BCE. NEO clips high and low temperatures, creating the spike on the right edge.

We can calculate the primary productivity diversity using the same method as for temperature. Rainfall, however, requires a slightly altered definition. Diamond emphasizes that the amount of rain does not matter as much as when in the year it falls. In particular, he argues that certain crops that were bred for summer rains can have difficulties growing in regions where there are winter rains, and vice versa. To capture this, we can calculate the standard deviation of rainfall levels in each month, leaving us with a 12-dimensional vector representing the monthly rainfall diversity. If one part of a society sees summer rains, while another sees winter rains, then the standard deviations representing summer and winter months will be high, because in one month the society will see both high and low amounts of rain in different regions. In order to characterize the overall rainfall diversity, we can then sum the elements of this vector.

\subsection{Comparisons}

We now have a measure of climate diversity, but this measure is different for each society. That is, with our current measure, we cannot compare the climate diversity of separate societies because some societies might be more or less diverse by virtue of their location or land area. For example, 
we would expect Russia to have a more diverse climate than Switzerland simply because it is much larger.

In order to assess whether a society grew so as to have a uniform climate, we can compare its climate to an approximation of the climate diversity that it gets from its land area and location. For each society, consider a disk with the same land area and centered on the same point (Figure 7). If the society had grown so as to have a uniform climate, we would expect its climate to be more uniform than that of its associated disk.

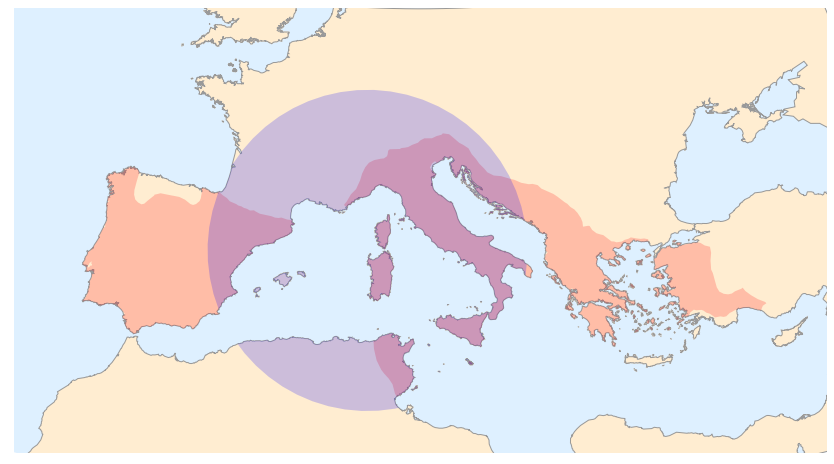

Figure 7: The red-tinted region shows the borders of the Roman Republic in the year $129 \mathrm{BCE}$. The blue-tinted region is generated by taking a disk and removing oceans. The disk is selected so that the blue-tinted region has the same land area as the red-tinted region, and is centered on the same point.

It is important to note that we want these disks to have the same land area as their associated societies, and so significant bodies of water should be removed. We can use numerical minimization to find disk radii that make land area within the disks are equal to the land area of their associated societies.

We can divide the climate diversity of a society's disk by the climate diversity of the society itself to get a measure of relative climate uniformity. This gives us a ratio that we can handle in a similar way to the aspect ratios from the previous section.

\section{$3.3 \quad$ Results}

Because we defined relative climate uniformity in terms of climate diversity, and we have three types of climate diversity (temperature, primary productivity, and rainfall), we will have three types of climate uniformity. Table 1 shows the geometric mean and standard deviation of relative climate uniformity across all societies. The fact that the geometric means are less than 1 tells us that the disks had slightly more uniform climates than their associated societies, going against Diamond's argument.

\begin{tabular}{l||l|l} 
& Geometric Mean & Standard Deviation \\
\hline Temperature & 0.998 & 0.0980 \\
Primary Productivity & 0.975 & 0.146 \\
Rainfall & 0.952 & 0.283
\end{tabular}

Table 1: Statistics of relative uniformities for all societies considered.

The fact that the standard deviations are so low tells us that individual disks performed very similarly to their associated societies. This might suggest that our definition of climate diversity always returns results in a small range. However, by looking at the distribution of temperature diversity across all societies (Figure 8), we see that our definition gives a wide range of values, suggesting that the similarities between the disks and their societies are not simply a product of how we defined climate diversity. 


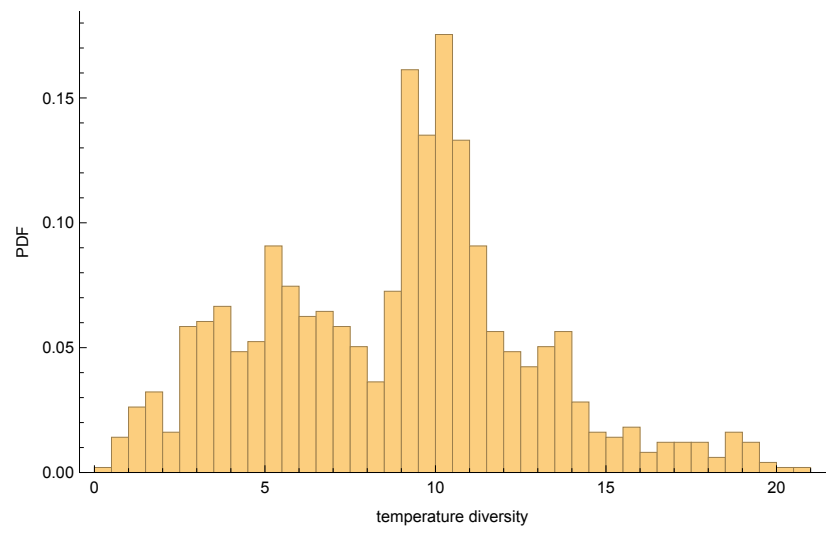

Figure 8: Distribution of temperature diversities, illustrating variability in temperature diversities.

Finally, just as with aspect ratios, Diamond's argument applies primarily to geographically large societies. However, Figure 9 shows that relative climate uniformity does not increase with land area, as Diamond's theory would suggest.
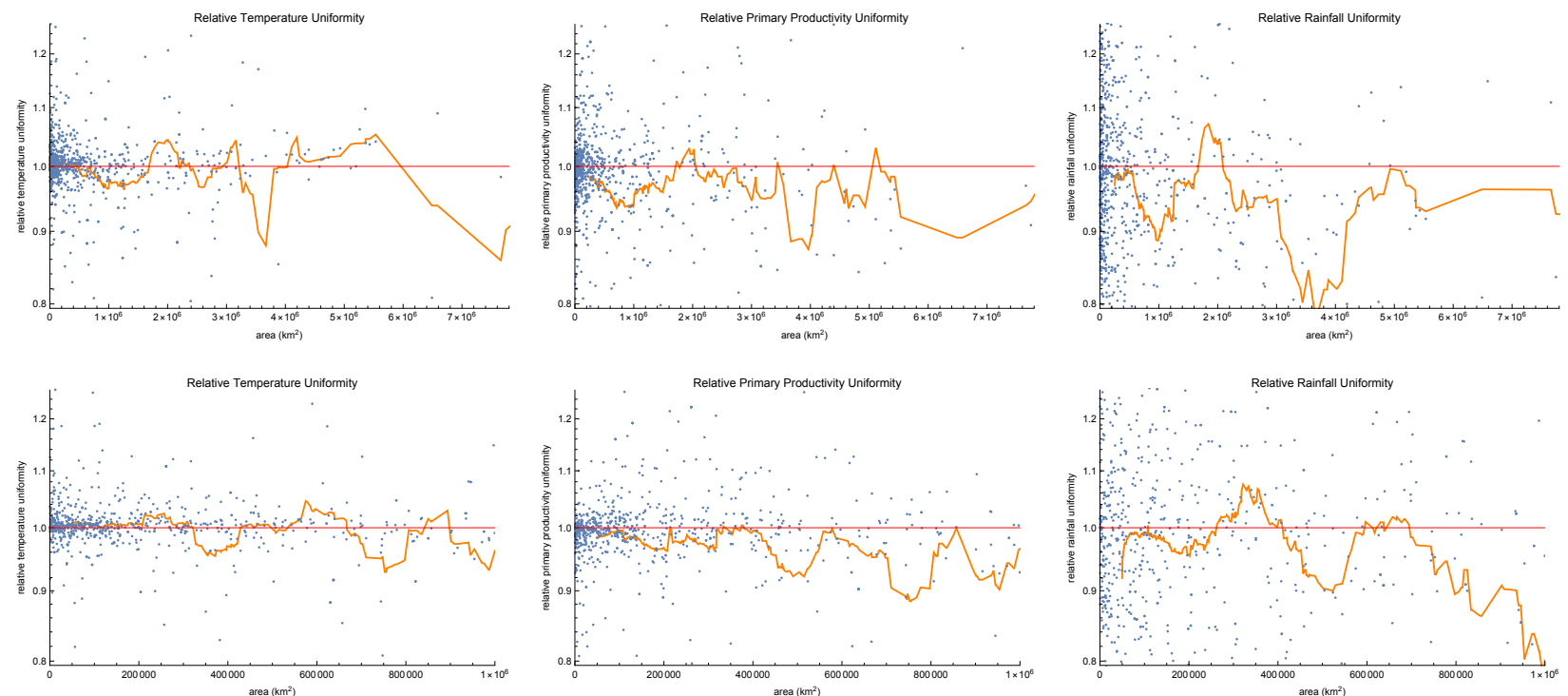

Figure 9: Relative uniformity of three climate variables as a function of area. The blue dots represent societies and the orange lines represent moving geometric means. The bottom row shows a smaller domain on the $\mathrm{x}$-axis than the top row. The moving means on the bottom row have a span of $100000 \mathrm{~km}^{2}$, while those on the top row have a span of $500000 \mathrm{~km}^{2}$.

\section{Conclusion}

Diamond makes two major claims: that societies are on average wider than they are tall, and that societies grow faster into regions with similar climates to their own.

To test the first claim, we started by characterizing aspect ratio. There is no single definition of aspect ratio on a globe, but we developed a moment of inertia based method that is projection independent and insensitive to outliers. With this method, we found that societies are on average wider than they are tall, agreeing with Diamond's claim. However, by Diamond's argument, we 
would expect larger societies to exhibit a larger effect, but this is not the case, suggesting another cause. We hypothesize that the orientation of coastlines might be a bigger effect, overshadowing the effects of climate.

In order to test Diamond's second claim, we used modern satellite climate data on temperature, primary productivity, and rainfall to characterize climate diversity. We created a measure of relative climate uniformity by comparing the climate diversity of each society to a disk of equal land area. We found that, on average, societies have extremely similar climate diversities to their associated disks and the effect is no larger with societies with higher land area, going against Diamond's claim.

\section{Acknowledgments}

I would like to thank Jared Diamond both for his work that directly led to this project, and for his encouragement based on an early draft of this paper. 


\section{References}

[1] Jared M. Diamond. Guns, Germs, and Steel: The Fates of Human Societies. W.W. Norton \& Company, 1998.

[2] Peter Turchin, Jonathan Adams, and Thomas Hall. East-west orientation of historical empires and modern states. Journal of World-Systems Research, 12(2):219-229, 2006.

[3] David D. Laitin, Joachim Moortgat, and Amanda Lea Robinson. Geographic axes and the persistence of cultural diversity. Proceedings of the National Academy of Sciences, 109(26):1026310268, 2012.

[4] Steven W Running, Ramakrishna Nemani, Joseph M Glassy, and Peter E Thornton. MODIS daily photosynthesis (PSN) and annual net primary production (NPP) product (MOD17). Apr 1999. 